\title{
Artificial embolization of carotid-cavernous fistula with post-operative patency of internal carotid artery
}

\author{
FABIAN ISAMAT, V. SALLERAS, AND A. M. MIRANDA \\ From the Services of Neurosurgery and General Surgery, Hospital de N. S. del Sagrado Corazón, Barcelona, \\ Spain
}

SUMMARY This report deals with a patient of 86 who developed a carotid-cavernous fistula. Artificial embolization alone was considered the safest treatment for this patient and proved to be adequate. Post-operative preservation of the patency of the internal carotid artery was demonstrated by angiography. We believe this method is particularly appropriate for carotid-cavernous fistulas if it is demonstrated by angiography that the major blood flow of the carotid artery pours into the fistula. A soft-iron clip attached to the muscle can be used for external and forceful guidance of the embolus into the fistula with the help of an electromagnet, hence the patency of the internal carotid artery can be preserved. The embolus should be introduced through the external carotid artery. This is the only case known to us in which patency of the internal carotid artery was post operatively maintained. We have reviewed 545 reported cases of surgically treated carotid-cavernous fistulas and analysed the results from simple cervical carotid ligation to the more sophisticateథ methods of artificial embolizations. The results obtained by artificial embolization have beep consistently good, while the other techniques have failed in large percentages. Artificial embolization should be used as the primary treatment for carotid-cavernous fistula, since ligation of the internal carotid artery precludes its embolization at a later date.

Because of the dramatic and intense disability produced by a carotid-cavernous fistula this intracranial vascular pathology has had considerable surgical attention in spite of the low rate of incidence. A variety of surgical techniques have been developed but most of the surgical approaches have dealt with a distant reduction of the blood flow into the fistulous tract rather than with the closure of the fistula itself. Examples are the single or combined ligation of the carotid arteries in the neck and isolation of the fistula between an intracranial carotid clip and a cervical carotid ligation, the so-called 'trapping' operation.

The first attempt to treat a carotid-cavernous fistula with a physiological approach to the problem was that of Brooks (1930) who successfully performed a muscle embolization of the intracavernous portion of the carotid artery. Since then a limited number of artificial embolizations with surgical (Hamby and Gardner, 1933; Gurdjian, 1938; Röttgen, 1948; Jaeger, 1949; Jaeger, 1959; SunderPlassmann and Tiwisina, 1952; Hamby, 1964;
Hamby, 1966; Kosary, Lerner, Mozes, and Lazar, 1968; Arutiunov, Serbinenko, and Shlykov, 1968) or accidental (Lang and Bucy, 1965) occlusion of the internal carotid artery in the neck, preceded in some with an intracranial carotid clipping, have been reported. Nonetheless all of these reported cases had a reduction of the ipsilateral carotid flow either due to the internal carotid ligation following the embolization or to post-operative carotid thrombosis.

This report describes the surgical treatment of a carotid-cavernous fistula in an 86 year-old woman who was too feeble to attempt an intracranial operation and who did not tolerate carotid occlusion. Successful regression of her pulsating exophthalmos with post-operative preservation of the internal carotid artery patency was obtained by intraluminal muscle embolization.

CASE REPORT

An 86 year-old woman was referred to the Service of Neurosurgery in February 1969 complaining of left-sided 
exophthalmos with ocular pain and progressive visual failure. She had sustained a minor left frontal injury when bumping the head against a door seven months before. On the following day she began to hear a bruit over the left eye that progressively increased in intensity and had remained constant since then. By the fourth day the patient complained of left retro-ocular pain and deterioration of visual acuity. Proptosis and ophthalmoplegia of the left eye rapidly followed in one week and her symptoms continued to increase up to the time of admission to hospital.

Examination revealed a small and frail old woman in acute distress. Her blood pressure was $180 / 100 \mathrm{~mm} \mathrm{Hg}$ and the pulse rate about 60 per minute and arrhythmic. She had a reduced respiratory capacity with an overall cyanotic discolouration, particularly intense in her lips.
There was severe dilatation of the left supraorbital veins. She had a nearly complete ophthalmoplegia with striking chemosis and conjunctival injection (Fig. 1). Direct or even distant auscultation of the left orbit revealed an intense bruit synchronous with the pulse. Her vision on the left side was reduced to appreciation of light and shadows. The results of the rest of the neurological examination were within normal limits except for absent vibration sensitivity in the lower extremities.

Plain radiographs of the skull were normal and left carotid angiography was performed under local anaesthesia. This showed a carotid-cavernous sinus fistula with striking dilatation of the ophthalmic and supraorbital veins and minor filling of the cerebral arteries (Fig. 2a, b). The angiography was well tolerated.

Digital carotid compression was performed during

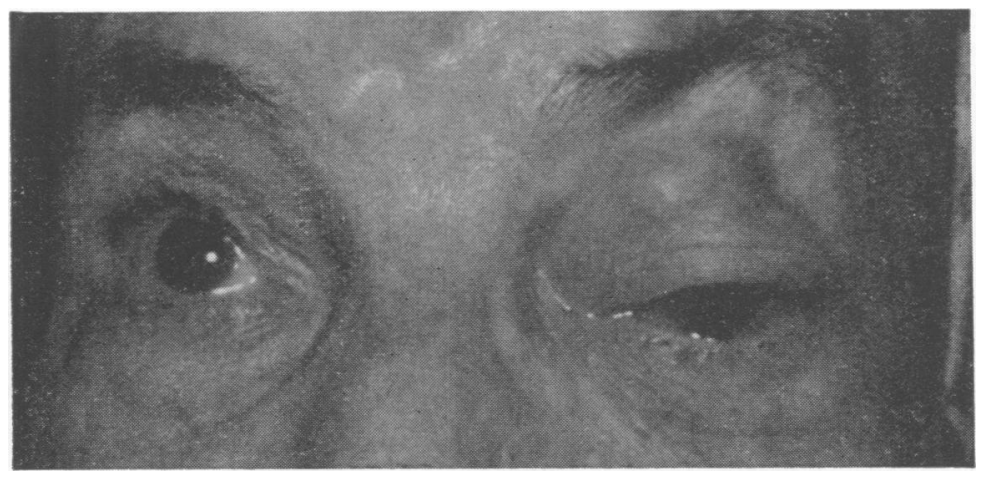

FIG. 1. Pre-operative photograph of ophthalmoplegia and exophthalmos of the left eye.

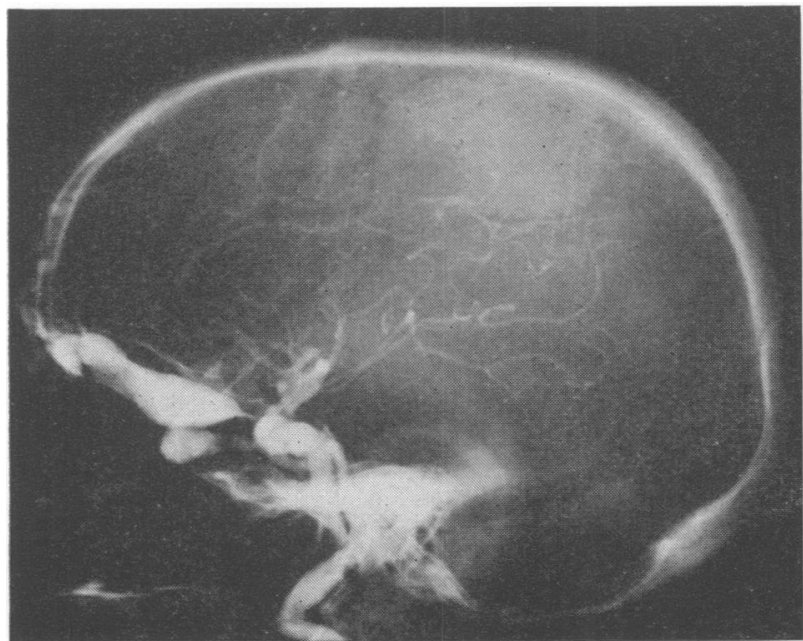

(a)

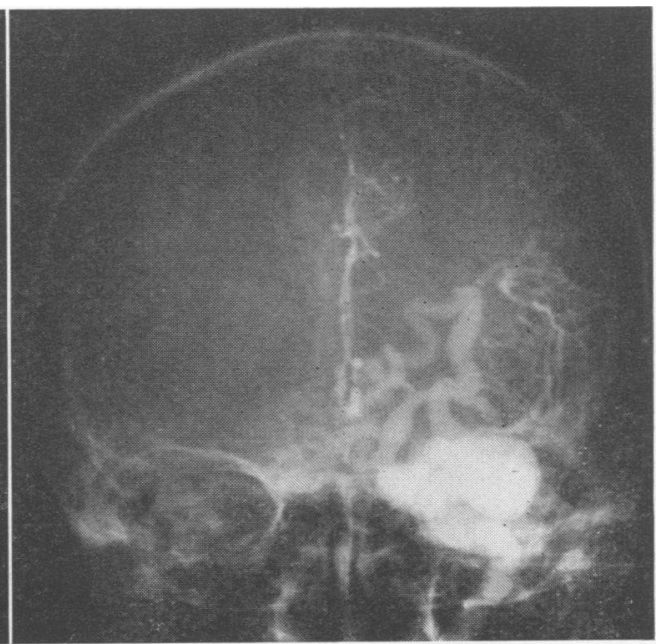

(b)

FIG. 2. Pre-operative left carotid angiograms. a. Lateral view, arterial phase, showing the immediate filling of the cavernous sinus and the ophthalmic veins and the diminished circulation of the cerebral arteries. b. Anterior projection with demonstration of the striking injection of the cavernous sinus and facial veins. 
EEG recording, revealing an almost complete intolerance to the compression with dysphasia and flattening of the tracing. Thus carotid ligation was considered too risky. An intracranial approach was also too dangerous as the surgical treatment for this old lady. Since by angiography most of the contrast was seen entering the fistula and only a minimal part into the cerebral arteries, artificial embolization was considered the safest procedure.

OPERATION Under local anaesthesia, the left common, internal, and external carotid arteries were isolated in the neck. After double ligation and section of the superior thyroid and the lingual arteries, ductus clamps were placed on the external carotid artery right at its origin and $3 \mathrm{~cm}$ further up. A small arteriotomy was done between the clamps. After the placement of ductus clamps on the common carotid artery and on the internal carotid artery, the proximal external carotid artery clamp was removed and a $1 \times 0.5 \mathrm{~cm}$ piece of omohyoid muscle with a clip attached to it was introduced into the arteriotomy. This artificial embolus was pushed into the common carotid artery and the proximal external carotid artery clamp was replaced. The clamps were removed from the internal and common carotid arteries and the muscle was forcefully propelled by the $180 \mathrm{~mm} \mathrm{Hg}$ of systolic pressure. Immediately the patient stated that the bruit had disappeared and it was not heard by auscultation. By TV monitoring we could see the clip right on the anterior floor of the sella turcica. Post-operative angiography was then performed and it demonstrated a complete disappearance of the fistulous tract with patency of the internal carotid artery and correct filling of the cerebral arteries (Fig. 3). The left external carotid artery was then ligated and the clamps were removed.

The immediate post-operative course was uneventful. The bruit and the retro-ocular pain remained absent.
Her cerebral condition was normal without sensorimotor or dysphasic deficits. By the second day the exophthalmos and the chemosis were receding, but no improvement was observed in her visual acuity. On the sixth post-operative day the movements of the left eye had returned to normal, although mild impairment of elevation of the upper eyelid was still present (Fig. 4). She was then up and about. Ten days after surgery she developed right pulmonary congestion with aggravation of the preoperative cardiac and respiratory symptomatology, and a complete right bundle branch block was recorded on electrocardiography. She was free of ocular complaints and of neurological deficits. The patient was discharged from the Neurosurgical Service and transferred to the cardiac-respiratory unit where she remained until her death from heart failure. No necropsy permission was obtained.

\section{DISCUSSION}

The most widely used treatment for carotidcavernous fistulas has been occlusion of the carotid artery in the neck. In a review of 545 reported cases of surgically treated carotid-cavernous fistulas we found that 330 had cervical ligation either of the internal carotid or of the common carotid arter or of both. Yet the 'cures' obtained were only 31 . to $40 \%$ depending on the chosen artery. Further $\frac{\rho}{\mathrm{N}} \mathrm{O}$ more, this operation deprives the ipsilateral cerebraq hemisphere of blood by the stealing reflux down the internal carotid artery and the suction of the stilg patent carotid-cavernous fistula.

A more aggressive procedure has been the opera tion of 'trapping' the carotid-cavernous fistula in

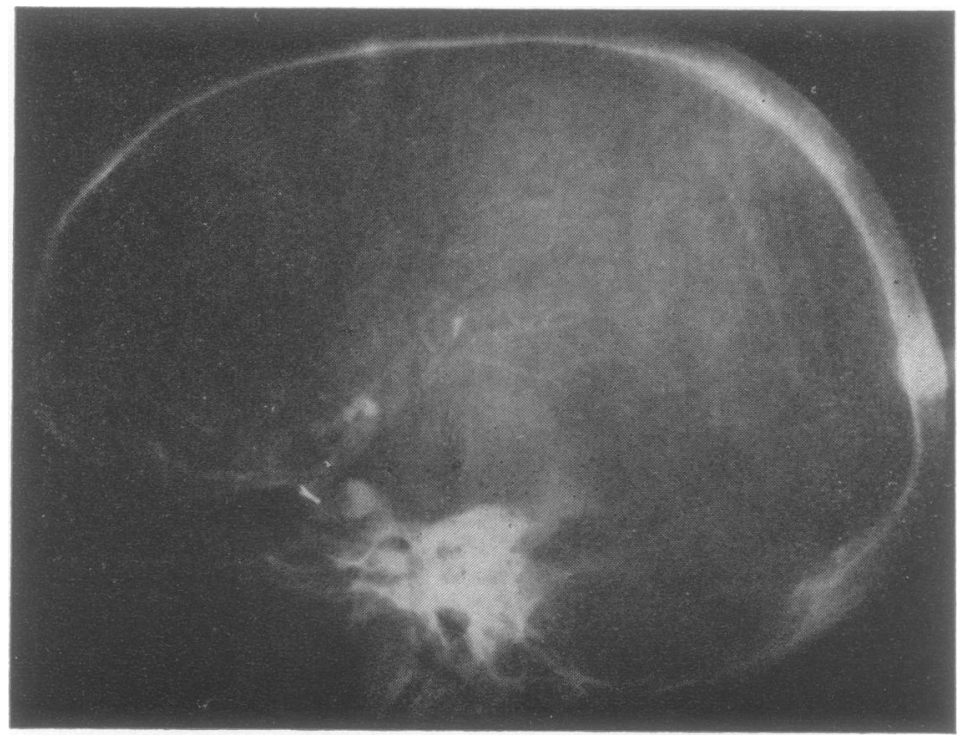

FIG. 3. Post-operative left carotid angiogram: the cavernous sinus is not filled by contrast medium. Note the clip attached to the muscle embolus closing the fistulous opening. There is patency of the internal carotid artery and its cerebral branches. 


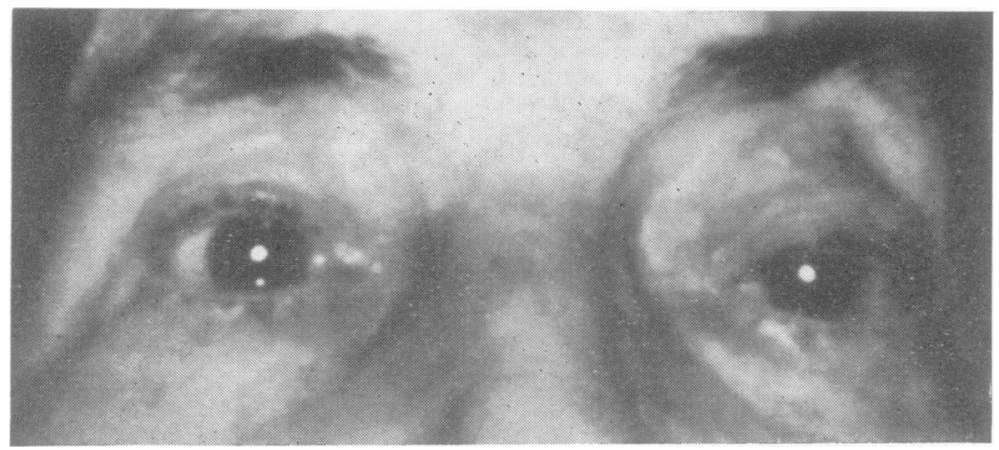

FIG. 4. Post-operative photograph showing the reduction of the ophthalmoplegia and the exophthalmos.

combined intracranial carotid artery clipping and internal carotid artery ligation in the neck, with $56.7 \%$ of good results. A complete 'trapping' involving the intracranial occlusion of the carotid and ophthalmic arteries followed by cervical ligation of the internal and external carotid arteries has been reported in 76 patients with satisfactory results in $78.8 \%$. The cause of failure is again that by reducing the blood flow to the fistula by a distant attack permits reverse collateral circulation and the presence of intracavernous anastomoses between the internal and the external carotid arteries (Hayes, 1963).

As stated by Brooks (1930), 'any successful operative method for the cure of arteriovenous fistula must have as its primary object the closure of the fistulous opening and not the limitation of the blood flow through the segment of artery containing the fistula'. Thus a direct approach to the intracavernous portion of the carotid artery would be ideal. This surgical rationale has been done successfully (Parkinson, 1965; Riechert, 1968) but at the present time it carries a tremendous surgical risk.

Another way to handle the problem would be the closure of the fistulous opening by an intraluminal approach. Brooks (1930) conceived this approach by inserting an artificial muscular embolus into the cervical carotid artery followed by arterial ligation. Lang and Bucy (1965) successfully repeated this technique but without ligation of the internal carotid artery, although it was followed by thrombosis of this artery. Kosary et al. (1968) used porcelain beads as the embolic material in one patient but again they ligated the cervical internal carotid artery.

We have found 89 other cases of artificial embolization. Seventy-six of them had the operation advised by Hamby (1964) to avoid the possibility of a cerebral embolic complication-namely, intracranial carotid artery clipping followed by cervical carotid embolization with muscle and carotid artery ligation. The cure rate was $98.6 \%$ but one patient died as result of the procedure. To eliminate the intracranial clipping, Arutiunov et al. (1968) developed their technique consisting of the cervical intraluminal placement of a clipped muscle embolus attached to a nylon string. The muscle is allowed to ascend under $x$-ray control to the intracavernous portion of the carotid artery and then secured in place by anchoring the nylon string to the internal carotid artery ligation. This was performed in 13 patients with $100 \%$ of adequate results.

Nonetheless, all the above operations included deliberate or accidental occlusion of the internal carotid artery. But the ideal treatment would be one that closes the fistula without interfering with the lumen of the internal carotid artery.

In our patient a cervical carotid artery ligation was obviously not going to succeed, since simple compression of the carotid artery was not well tolerated. Hence the Arutiunov operation was discarded. The age and physical condition of the patient discouraged us from doing an intracranial clipping by Hamby's technique. It was necessary that the treatment be as simple as possible. Since the angiographic studies demonstrated that most of the contrast medium was taken up by the fistula and only a minimal part passed into the cerebral arteries, it was thought that a small embolus would follow the same dynamic pattern and could successfully plug the fistula.

Sunder-Plassmann and Tiwisina (1952) pointed out the risk of cerebral emboli if the major carotid flow did not pour into the fistula. That was not the case in our patient. Therefore embolization with muscle was considered the treatment of choice. For maximum preservation of constant internal carotid artery flow the embolus was introduced through the external carotid artery. The size of the muscle was considered to be small enough to go through the fistulous opening and large enough to be trapped between the cavernous sinus trabeculations. We also thought of guiding a previously magnetized clip 
attached to the embolus by an electromagnet over the skin covering the supero-anterior part of the left zygoma. However, this was not needed in this case, since the great differential pressure between the arterial pressure and the venous pressure of the cavernous sinus was enough properly to conduct the embolus and plug the fistulous opening in its cavernous side. In this way the patency of the internal carotid artery was not interfered with as was later demonstrated by carotid angiography.

We believe that artificial embolization is the treatment of choice for carotid-cavernous fistulas. If most of the blood flow as demonstrated by carotid angiography follows the fistulous tract, simple embolization without carotid ligation can be done safely and successfully. A magnetized and insulated soft-iron clip applied to the muscle can be used as an x-ray mark as well as for forceful external electromagnetic guidance. It is important to introduce the embolus through an external carotid arteriotomy, not just to preserve the lumen of the internal carotid artery but to avoid unnecessary handling and prolonged temporal occlusion of this artery.

\section{REFERENCES}

Arutiunov, A. I., Serbinenko, F. A., and Shlykov, A. A. (1968). Surgical treatment of carotid-cavernous fistulas Progr. Brain Res., 30, 441-444.

Brooks, B. (1930). Discussion of Noland, L., and Taylor, A. S. Pulsating exophthalmos, the result of injury. Trans. Sth. surg. Ass., 43, 176-177.

Browder, J. (1937). Treatment of carotid artery-cavernous sinus fistula. Report of a case. Arch. Ophthal., 18, 95-102.
Gurdjian, E. S. (1938). Packing of internal carotid artery with muscle in treatment of carotid-cavernous arteriovenous aneurysm. Arch. Ophthal., 19, 936-940.

Hamby, W. B. (1964). Carotid-cavernous fistula. Report of 32 surgically treated cases and suggestions for definitive operation. J. Neurosurg., 21, 859-866.

Hamby, W. B. (1966). Carotid-Cavernous Fistula. Thomas: Springfield, Ill.

Hamby, W. B., and Gardner, W. J. (1933). Treatment of pulsating exophthalmos with report of two cases. Arch. Surg., 27, 676-685.

Hayes, G. J. (1963). External carotid-cavernous sinus fistulas. J. Neurosurg., 20, 692-700.

Jaeger, R. (1949). Intracranial aneurysms. Sth Surg., 15, 205-217.

Jaeger, R. (1959). In discussion of paper by D. H. Echols and J. D. Jackson. Carotid-cavernous fistula: a perplexing surgical problem. J. Neurosurg., 16, 626.

Kosary, I. Z., Lerner, M. A., Mozes, M., and Lazar, M. (1968). Artificial embolic occlusion of the terminal internal carotid artery in the treatment of carotid-cavernous fistula. J. Neurosurg., 28, 605-608.

Lang, E. R., and Bucy, P. C. (1965). Treatment of carotidcavernous fistula by muscle embolization alone. The Brooks method. J. Neurosurg., 22, 387-392.

Parkinson, D. (1965). A surgical approach to the cavernous portion of the carotid artery. Anatomical studies and case report. J. Neurosurg., 23, 474-483.

Pool, J. L., and Potts, D. G. (1965). Aneurysms and Arteriovenous Anomalies of the Brain: diagnosis and treatment? Hoeber: New York.

Riechert, T. (1968). A new surgical method for treatment of pulsating exophthalmos. Progr. Brain Res., 30, 445-449.

Röttgen, P. (1948). Zur Behandlung der Carotis-SinuQ cavernosus-Aneurysmen. Langenbecks Arch. klin. Chir. 207, 613-633.

Sunder-Plassmann, P., and Tiwisina, T. (1952). Die Behand lung der Aneurysmen in Sinus Cavernosus (Exophthalmu pulsans). Chirurg, 23, 376-382.

Tönnis, W. (1960). Zur Entstehung der Rezidive bei de몰 Behandlung der Carotis-Sinus-Cavernosus-Aneurysmen und ihre Verhütung. Langenbeck's Arch. klin. Chir., 295, 186-191. 